\title{
Técnica de Recomendação Baseada em Metadados pa- ra Repositórios Digitais Voltados ao Ensino
}

\author{
A Recommendation Technique Based on Metadata for Digital Repositories Oriented \\ to Learning
}

\author{
Marcus Filipi Rosso Casagrande \\ PPGCC - UFSC \\ CP 476 \\ 88040-900 Florianópolis \\ marcus.xj220@gmail.com
}

\author{
Gustavo Kozima da Silva \\ UFSC \\ CP 476 \\ 88040-900 Florianópolis \\ gukozima@gmail.com
}

\author{
Roberto Willrich \\ PPGCC - INE - UFSC \\ CP 476 \\ 88040-900 Florianópolis \\ roberto.willrich@ufsc.br
}

\begin{abstract}
Resumo Repositórios Digitais (RDs) estão sendo cada vez mais utilizados na área de ensino para organizar e disponibilizar coleções digitais de objetos de aprendizagem. Este artigo propõe uma técnica de recomendação simples e escalável aplicada a RDs, que visa apoiar os usuários na localização de conteúdos relevantes. Na técnica proposta, as preferências dos usuários são estimadas implicitamente via a observação dos valores de alguns metadados dos conteúdos acessados pelos usuários. Visando aumentar a escalabilidade, na técnica proposta os usuários são agrupados em grupos de interesses, reduzindo o espaço de busca na filtragem colaborativa. A técnica proposta foi testada e avaliada em um RD utilizado no ensino de literatura. Os resultados demonstram a efetividade da técnica ao menos do dominio de ensino de literatura.
\end{abstract}

Palavras-Chave: Sistemas de Recomendação, Repositórios Digitais.

\begin{abstract}
Digital Repositories (DRs) are being increasingly used in the field of education for organize and make available digital collections of learning objects. This paper proposes a simple and scalable recommendation technique applied to DRs for supporting users in finding relevant contents. In the proposed technique, the users preferences are implicitly estimated by observing some metadata values of the accessed content. To increase the scalability, in the proposed technique the users are grouped in interest groups to reduce the search space in the collaborative filtering. The proposed technique is tested and evaluated in a DR used for literature teaching. The experiments show the effectiveness of the technique at least in the domain of literature teaching.
\end{abstract}

Keywords: Recommendation Systems, Digital Repositories. 



\section{Introdução}

Repositórios digitais (RD) são sistemas, geralmente Web, que oferecem recursos para organizar coleções digitais e para construir um catálogo dos metadados. No contexto educacional, as coleções dos RDs são formadas por conteúdos, ou objetos, de aprendizagem. Por sua vez, o catálogo de metadados oferece suporte à organização e à descoberta de conteúdos digitais. A recomendação é que os RDs adotem algum padrão aberto de esquema de metadados, como IEEE LOM [1] ou Dublin Core [2], responsável pela descrição dos recursos disponibilizados.

$\mathrm{Na}$ literatura, diversos termos são empregados para referenciar um repositório de conteúdos digitais, como Repositório Digital (RD), Biblioteca Digital ou Biblioteca Virtual. Este trabalho considera que Bibliotecas Digitais ou Virtuais são repositórios de conteúdos construídos sobre os princípios rígidos de gerenciamento de informação aplicados em biblioteconomia por centenas de anos. $\mathrm{RD}$ é adotado neste trabalho como um termo genérico, que é usado para referenciar sistema que, além das Bibliotecas Digitais, engloba repositórios que não se baseiam em técnicas rígidas de gerenciamento da informação.

A Biblioteca Digital de Literatura Brasileira (BD-LB) (www.literaturabrasileira.ufsc.br) é um exemplo de RD voltado ao ensino. Este RD disponibiliza uma grande coleção de obras literárias e outros conteúdos relevantes ao ensino de literatura, incluindo um banco de dados de autores brasileiros e portugueses. A BD-LB vem sendo utilizada em diversas atividades de ensino de Literatura da Universidade Federal de Santa Catarina (UFSC).

Apesar dos RDs oferecerem meios para a organização dos conteúdos, com o aumento das coleções, o usuário se depara com o problema clássico de sobrecarga de informação. Nesta situação, muitas vezes os usuários dos RDs realizam um grande esforço para localizar a informação desejada dentro de um conjunto enorme de informações disponíveis. Portanto, é importante adicionar funcionalidades aos RDs que permitam a localização mais eficiente de conteúdos. Uma alternativa para isto é o uso dos chamados sistemas de recomendação, que apoiam os usuários na localização de conteúdos através da apresentação de uma lista de conteúdos relevantes, que são potencialmente interessantes ao usuário.

Este artigo descreve uma técnica simples e eficiente de recomendação de conteúdos para RDs [3]. Nesta técnica, o perfil do usuário é construído implicitamente pelo sistema, observando os valores de alguns dos metadados dos conteúdos acessados. Esta construção implícita do perfil evita uma sobrecarga de trabalho do usuário para expressar suas preferências.
A redução da complexidade da técnica de recomendação proposta é alcançada pela utilização de valores dos metadados dos conteúdos acessados, não exigindo técnicas complexas de análise destes conteúdos. O agrupamento de usuários e itens é outra técnica adotada para reduzir o tempo de processamento necessário à recomendação.

A técnica de recomendação proposta foi implementada na BD-LB, com a qual foram realizados experimentos com usuários reais com o objetivo de avaliar a técnica proposta. Os resultados demonstram a efetividade da técnica proposta, pelo menos para RDs aplicados no ensino de literatura.

O restante deste artigo está organizado na forma que segue. A Seção 2 revisa conceitos gerais sobre sistemas de recomendação. A Seção 3 apresenta os trabalhos relacionados na área de recomendação de livros e objetos de aprendizagem. A Seção 4 apresenta a técnica de recomendação proposta. Na sequência, a Seção 5 descreve a implementação da técnica proposta e apresenta os resultados dos testes realizados, em termos de cobertura, precisão e tempos de resposta. Finalmente, as conclusões e trabalhos futuros são apresentados na Seção 6.

\section{Sistemas de Recomendação}

Um sistema típico de recomendação procura identificar dentro de um conjunto de itens (conteúdos, produtos ou serviços) aqueles que possam ser de interesse de um determinado usuário. Este tipo de sistema é útil quando o universo de escolha é muito grande e é possível realizar uma estimativa das preferências dos usuários.

Diversas técnicas de recomendação já foram propostas e aplicadas em diversos domínios. Em [4] a [7], os autores revisam os principais conceitos, técnicas e algoritmos utilizados pelos sistemas de recomendação e apresentam as suas particularidades e objetivos para cada domínio de aplicação.

No segmento de e-commerce, por exemplo, os sistemas de recomendação são utilizados para facilitar a localização de produtos/serviços e com isso aumentar a rentabilidade. Além de serem utilizados para recomendar produtos em e-commerce, os sistemas de recomendação vêm sendo utilizados para recomendar conteúdos, como artigos científicos, músicas, filmes e programas de TV.

Dois domínios são particularmente importantes no contexto deste trabalho: a recomendação de livros e de conteúdos educacionais. Convencionalmente, as técnicas de recomendação utilizadas em e-commerce também abrangem livros, como qualquer outro produto a venda. Em geral, a recomendação de livros utiliza informações dos livros, ou seja, seus metadados, como gênero, auto- 
res, ano de publicação, palavras-chave, etc ([8], [9]).

No contexto educacional, os sistemas de recomendação vêm sendo aplicados com o objetivo de buscar e filtrar conteúdos educacionais relevantes a alunos e professores ([10] e [11]). Em [5], os autores demonstram o potencial dos sistemas na recomendação de recursos digitais educacionais.

De maneira geral, e independente do domínio que são aplicadas, as técnicas de recomendação consideram: (i) as informações sobre o conjunto de itens (conteúdos, produtos ou serviços) a serem recomendados; (ii) as informações sobre os usuários do sistema; e (iii) a técnica que a partir de (i) e (ii) gera a lista de itens recomendados para um dado usuário.

\subsection{Informações sobre os itens a recomendar}

As informações sobre o conjunto de itens a recomendar podem ser obtidas de diversas formas, dependendo da técnica de recomendação adotada. Muitos sistemas utilizam o conjunto de metadados que descrevem cada item, como título, autor, gênero, palavras-chave, etc. Outras técnicas extraem informações do próprio conteúdo a partir da análise automática do conteúdo dos itens (como indexação de frequência de termos).

\subsection{Informações sobre os usuários}

Muitas técnicas de recomendação se baseiam na construção de um modelo ou perfil de usuário, que mantém dados que representam as preferências e interesses de um determinado usuário. Este perfil mantém as informações necessárias para estimar o grau de interesse (ou de utilidade) do usuário sobre um item.

O perfil do usuário pode ser construído com a ajuda explícita dos usuários, onde eles oferecem ao sistema seus dados demográficos (como idade, sexo, nível educacional, localização geográfica) e suas preferências/necessidades. Em muitas técnicas, o usuário deve inclusive realizar avaliações dos itens acessados/comprados. A desvantagem desta construção explícita do perfil é a necessidade do usuário se dar ao trabalho de preencher as informações requeridas. Diversos trabalhos sinalizam que os usuários tendem a não oferecer este tipo de informação [12].

Este problema não ocorre em técnicas de recomendação que adotam a construção implícita do perfil do usuário, sem necessidade de intervenção direta do usuário. Nestas técnicas, as preferências e interesses dos usuários são estimados com base nas interações do usuário com o sistema, observando, por exemplo, os conteúdos acessados, os critérios de consultas e/ou histórico de navegação.

\subsection{Técnicas de recomendação}

Em [13], o autor distingue cinco principais classes de técnicas de recomendação: Filtragem Colaborativa (FC), Filtragem Baseada em Conteúdo (FBC), Filtragem Demográfica, Filtragem Baseada em Utilidade e Filtragem Baseada em Conhecimento.

As técnicas mais adotadas são baseadas na FC e FBC. Na FBC, o perfil do usuário mantém a lista de itens já acessados/comprados por ele e o grau de interesse, ou satisfação, do usuário por cada um destes itens. Este grau de interesse é geralmente informado explicitamente pelo usuário, na forma de uma avaliação positiva ou negativa do item. Com base nas informações sobre os itens (Seção 2.1), o sistema recomenda itens que são similares àqueles que o usuário avaliou positivamente. Por exemplo, no caso do uso de metadados, o sistema pode recomendar novos livros do gênero romance se o usuário avaliou positivamente obras deste gênero anteriormente.

A FBC possui algumas vantagens e desvantagens ([6], [7]). Uma das suas vantagens é que itens que não foram avaliados podem ser recomendados, possibilitando que todos os itens sejam passíveis de recomendação. Uma das desvantagens é a superespecialização, onde o sistema tende a gerar recomendações de itens muitos similares aos já acessados, e portanto, com um grau de novidade limitado. Outro aspecto crítico é a escalabilidade, caso o número de itens a serem analisados sejam muito grande.

Em geral, as técnicas de FC adotam um perfil mantendo o grau de interesse do usuário por cada item que ele já acessou. Este grau de interesse pode ser obtido pela avaliação explícita do item, ou então usando técnicas automáticas para estimar este grau de interesse. A partir do perfil de um usuário, os itens a serem recomendados a um usuário são identificados dentro do conjunto de itens já avaliados pelos "vizinhos próximos" deste usuário. Vizinhos próximos são usuários com perfis similares ao usuário foco da recomendação.

Técnicas de FC também possuem suas vantagens e desvantagens ([6], [7]). Uma das vantagens é evitar o problema da superespecialização e a possibilidade de identificar grupos de usuários com interesses comuns. Como desvantagens, pode-se citar os problemas de esparsidade e de escalabilidade. O problema da esparsidade se deve ao fato de que alguns conteúdos acessados por um usuário podem ter tido poucos (ou mesmo nenhum) acessos por parte dos usuários do sistema, dificultando a recomendação de conteúdos impopulares. A escalabilidade está relacionada à complexidade dos algoritmos de FC: geralmente a quantidade de informações a serem consideradas é tão grande que pode tornar o tempo de resposta um problema. 
Uma das principais técnicas utilizadas para melhorar a escalabilidade em sistemas de recomendação é o agrupamento (também chamado de clusterização) de usuários ou de conteúdos ([14], [15]). O objetivo é reduzir a quantidade de dados sobre o qual a FC será aplicada e com isto reduzir o tempo de processamento.

$\mathrm{Na}$ filtragem demográfica, o perfil do usuário mantém informações demográficas do usuário (idade, sexo, localização geográfica, etc.), e realiza recomendações baseadas nas classes demográficas. Por exemplo, o sistema pode recomendar restaurantes na cidade onde o usuário mora e mais adequados à faixa etária do usuário. Por sua vez, as técnicas de filtragem baseada em conhecimento recomendam itens baseado no conhecimento específico do domínio, incluindo como certas características dos itens satisfazem as necessidades e preferências dos usuários [13].

Alguns sistemas de recomendação são baseadas em técnicas hibridas, que combinam duas ou mais técnicas de recomendação visando melhorar o resultado e ao mesmo tempo tentando eliminar ou reduzir as desvantagens do uso de apenas uma única técnica.

\section{Trabalhos Relacionados}

Este trabalho apresenta uma técnica de recomendação para RDs, com a avaliação na área de literatura, em particular, na utilização destas obras no ensino. Esta seção analisa alguns trabalhos relacionados na área de recomendação de livros e técnicas de recomendação voltadas à área de ensino.

\subsection{Sistemas de Recomendação de Livros}

Existe um certo número de sites que recomendam livros com base em informações sobre livros e usuários. Dentre estes sites, openingthebook.com adota a FBC, utilizando informações fornecidas explicitamente pelo usuário para recomendar livros. Já whatshouldireadnext.com utiliza a FC, onde os usuários informam explicitamente os livros que gostam e o sistema, após identificar os vizinhos próximos, recomenda uma série de livros.

LibraryThing.com inclui um sistema de recomendação que utiliza uma técnica híbrida para recomendar livros. Para isso, ele utiliza diversas informações, como metadados dos livros, coleções de livros de cada usuário, avaliações destes livros e marcações (tags) realizadas pelos próprios usuários.

Conforme [16], a Amazon.com adota uma técnica de recomendação chamada de filtragem colaborativa itempara-item. Este algoritmo recomenda itens similares aos itens que o usuário comprou ou avaliou. A filtragem colaborativa ocorre aqui na descoberta de itens similares, que se baseia no princípio de "quem comprou o item também comprou junto ..." . Para tratar a escalabilidade e desempenho, a descoberta de itens similares é realizada em modo off-line e armazenada em uma tabela similar-items.

São poucos os detalhes técnicos oferecidos pelos sites de recomendação de livros citados anteriormente. Mas é possível avaliar as formas de construção do perfil do usuário. Neste aspecto, a Amazon.com realiza uma construção implícita e explícita do perfil do usuário, observando a compra dos usuários e analisando (se existir) as suas avaliações. Os demais se baseiam exclusivamente na construção implícita do usuário. Mas isto não pode ser vista como uma limitação destes sites, pois eles têm o propósito exclusivo de recomendar livros, e, portanto, os usuários não deveriam considerar um trabalho extra a construção de seu perfil. Já em sites de e-commerce, em geral o objetivo do usuário é localizar um produto para comprar, e a tarefa de avaliar os itens pode ser considerada incômoda pelo usuário.

Na literatura já existem algumas propostas de sistemas de recomendação para livros. Em [17], os autores propõem um sistema de recomendação híbrido, que combina a FC, FBC e filtragem demográfica. Este sistema adota uma técnica de clusterização de usuários, onde eles são agrupados considerando aspectos demográficos, como sexo e faixa etária. Segundo os autores, estes aspectos podem ser obtidos usando ferramentas de Web Scraping (extração automática de informações de sites Web). Esta técnica híbrida parece promissora, mas os autores não apresentam detalhes técnicos. Além disso, a FC e FBC se baseia em avaliações explícitas dos usuários.

O sistema de recomendação BibTip [18] é baseado na construção implícita do perfil do usuário. Ele observa o comportamento do usuário interagindo com um catálogo on-line (OPAC - Online Public Access Catalog), observando os acessos aos itens. Com base em uma análise estatística destes acessos, o sistema calcula coocorrências entre pares de itens (métrica quantificando o percentual de acessos de pares de itens em uma mesma sessão de navegação). A matriz de coocorrência é usada para gerar a recomendação. Nesta proposta, o problema de escalabilidade do sistema não é tratado.

Em [19], os autores propõem um sistema de recomendação para um RD. Esta técnica utiliza a FC onde os perfis dos usuários são extraídas implicitamente dos logs de acesso. Diferente da presente proposta, [19] não utiliza os metadados das obras para determinar preferências, apenas acessos às obras. Desta maneira, as características do livro não são consideradas.

\subsection{Sistemas de Recomendação no Ensino}

Já existem diversos trabalhos propondo técnicas de recomendação para RDs e voltadas especificamente para 
a área de ensino. Trabalhos como [20] e [21] propõem técnicas de recomendação para RDs baseadas em FC, mas ambas as propostas se baseiam na avaliação explícita dos conteúdos acessados. Esta é justamente uma das deficiências destas propostas, pois os usuários geralmente tendem a não oferecer este tipo de informação. Além disso, elas também sofrem dos problemas de esparsidade e escalabilidade associadas a FC.

Em [22], os autores propõem um modelo de recomendação de conteúdos educacionais descritos por metadados. Tal modelo visa capturar as informações do usuário implicitamente, utilizando o padrão de metadados OBAA [23]. A proposta mostra uma arquitetura interessante, que inclui elementos de Web Semântica no perfil do usuário e nos conteúdos disponíveis. Porém, este trabalho apresenta um modelo teórico, sem formalizar o procedimento realizado para a recomendação.

A abordagem para recomendação de objetos de aprendizagem e-LORS [24] adota a FBC e considera o relacionamento entre perfis e objetos de aprendizagem. Nesta proposta, o modelo de usuário mantém informações pessoais do usuário, seu perfil de aprendizagem (usando dimensões de preferências de aprendizagem de [25]), e suas restrições tecnológicas. Com base no perfil do usuário e nos metadados LOM, e-LORS realiza três etapas de filtragem: filtragem por conceitos (filtra os objetos a partir do tema buscado), filtragem por perfil de aprendizagem e filtragem por tecnologia (filtra os objetos que atendem às restrições de tecnologias do usuário). A filtragem por perfil de aprendizagem compara as preferências de aprendizagem com os Tipo de Interatividade e Tipo de Recurso de Aprendizagem da categoria Educacional do padrão LOM. Um aspecto interessante desta abordagem é a possibilidade de integração com ambientes virtuais de aprendizagem.

Em [10], os autores fazem uso de um sistema híbrido de recomendação de materiais didáticos, que combina a $\mathrm{FC}, \mathrm{FBC}$ e recomendação de conteúdos mais populares. Em tal proposta, o perfil do usuário é construído de forma explícita, onde os usuários do sistema devem informar suas áreas de interesse.

\subsection{Técnicas de Agrupamento}

Uma das formas de tratar o problema da escalabilidade dos sistemas de recomendação é a redução do espaço de busca via o agrupamento de usuários [14], ou de conteúdos [15]. O agrupamento visa reduzir a quantidade de informação sobre o qual a filtragem será realizada. O objetivo, portanto, é reduzir o tempo de processamento dos algoritmos de recomendação.

Existem muitas técnicas de agrupamento, sendo que a $k$-means [26] é a mais utilizada. Em [14] e [15], os auto- res propõem técnicas de recomendação que adotam a clusterização baseada na $k$-means. Nessa técnica $k$-means é definida inicialmente uma quantidade $k$ de clusters a serem criados e são escolhidos aleatoriamente $k$ dados (perfis de usuários ou itens) do conjunto a ser comparado e os colocam como centroide de cada cluster. Em seguida, os demais dados são distribuídos nos clusters, sendo que um dado é alocado a um cluster quando o dado é mais similar ao centroide do respectivo cluster. A cada ciclo, o centroide de cada cluster é atualizado como a média dos valores dos elementos do cluster.

Apesar da vantagem da redução do custo computacional, as técnicas de agrupamento podem gerar certa perda de precisão dos resultados, pois a busca do sistema de recomendação é limitada a um subgrupo específico, excluindo outras informações [14].

A proposta apresentada neste trabalho se destaca dos trabalhos descritos anteriormente pela junção de características que simplificam a complexidade de todo o processo de recomendação. Em particular, a construção do perfil do usuário é implícita, se baseando na análise dos valores dos metadados dos conteúdos acessados pelo usuário. Além disso, se definiu uma técnica de agrupamento que reduz o tempo de resposta da recomendação.

\section{Técnica de recomendação proposta}

A técnica de recomendação proposta para $\mathrm{RDs}$ segue uma abordagem híbrida, utilizando a FC e a FBC combinadas com uma técnica de agrupamento de usuários com perfis similares. A presente proposta assume a hipótese que os conteúdos acessados por grupos de usuários com perfis semelhantes possuem grande probabilidade de serem de interesse de um usuário individual pertencente a este grupo. Além disso, esta técnica é aplicável exclusivamente em RDs ou outros sistemas em que os conteúdos disponibilizados são descritos por um conjunto de metadados.

A técnica de recomendação proposta segue quatro etapas que serão descritas formalmente a seguir: (i) construção dos perfis dos usuários; (ii) agrupamento de usuários; (iii) determinação dos vizinhos próximos em cada grupo; e (iv) construção da recomendação (lista ordenada de conteúdos recomendados para o usuário).

\subsection{Definição do RD}

Para a formalização da técnica, é necessário primeiro definir formalmente RD. Para tal, considere a seguinte definição de RD (1), adaptada de Furtado [27].

$R D=(D, M, U, P U)$

onde: 
- $D=\left\{d_{i} \mid \mathrm{i} \in[1, \mathrm{I}]\right\}$ é o conjunto de documentos (conteúdos digitais) do $\mathrm{RD}$, sendo que I é o número total de conteúdos.

- $M=\left\{m_{j} \mid \mathrm{j} \in[1, \mathrm{~J}]\right\}$ é o conjunto de elementos de metadado adotados pelo $\mathrm{RD}$, sendo que $\mathrm{J}$ é o número total de elementos de metadado.

- $U=\left\{u_{k} \mid \mathrm{k} \in[1, \mathrm{~K}]\right\}$ é o conjunto de usuários registrados no $\mathrm{RD}$, sendo que $\mathrm{K}$ é o número total de usuários registrados.

- $P U=\left\{p u_{k} \mid \mathrm{k} \in[1, \mathrm{~K}]\right\}$ é o conjunto de perfis de usuários registrados no $\mathrm{RD}$, sendo que cada usuário $u_{k}$ tem um perfil $\mathrm{pu}_{\mathrm{k}}$.

\subsection{Perfil do Usuário}

O modelo de perfil de usuário adotado é similar ao adotado na técnica de recuperação personalizada de informações descrita em [27]. Formalmente, o perfil de um usuário $u_{k}$ é definido por (2).

$\mathrm{pu}_{\mathrm{k}}=\left(P E_{\mathrm{k}}, C A_{\mathrm{k}}\right)$

onde Preferências Específicas (PEs) são as preferências do usuário, e Conteúdos Acessados (CA) são os conteúdos acessados pelo mesmo usuário. As PEs de um usuário são estimadas implicitamente com base na análise dos valores dos Metadados Observados (MO) dos conteúdos acessados pelo usuário. Os MOs são definidos por (3).

$M O=\left\{m o_{n} \mid \mathrm{n} \in[1, \mathrm{~N}], N \leq J\right\}$

onde:

- MO é um subconjunto dos elementos de metadados observados (mo) para a construção do perfil do usuário;

- N é o número de elementos de metadados observados.

A seleção dos MOs depende do domínio do RD. Para tal, é necessário analisar quais metadados são relevantes para a determinação das preferências do usuário. Diferentes domínios possuem diferentes aspectos do conteúdo que são mais relevantes para identificar as preferências dos usuários. Para a recomendação de livros, [28] identifica alguns aspectos relevantes:

- Acessibilidade: a linguagem, tamanho ou o nível de dificuldade do livro;

- Conteúdo: o tópico, enredo, gênero, estilo, ou legibilidade de um livro;

- Engajamento: tipos afetivos de experiências de leitura evocados pelo livro;
- Familiaridade: livros similares a outros livros conhecidos ou relacionados com experiências anteriores;

- Item conhecido: Descrições dos livros são conhecidas, como título e/ou autor.

- Metadados: aspectos como título, autor, ano de publicação e formato;

- Novidade: livros que não são usuais ou peculiares, ou têm um conteúdo novo;

- Sociocultural: livros relacionados à experiência sociocultural do usuário, com um impacto cultural ou social particular, ou são populares ou obscuros.

Alguns aspectos listados dependem da identificação explícita do perfil do usuário, como familiaridade e aspectos socioculturais. Os demais aspectos citados por [28] poderiam ser representados por metadados em RDs. Mas infelizmente, muitos RDs adotam esquemas de metadados que descrevem basicamente os aspectos de metadados (título, autor, ano de publicação, formato, etc.), e poucos aspectos de conteúdo (tópicos, na forma de palavras-chave e gênero). A título de exemplo, os metadados disponíveis pela BD-LB descrevem apenas os aspectos de metadados. E dentre estes metadados, determinou-se, a partir de uma consulta aos profissionais da área de literatura, que os metadados autor e gênero literário são os metadados mais significativos para determinar se uma obra é relevante ou não para os usuários típicos. Naturalmente, outros metadados poderiam ser considerados no caso de professores e alunos, como por exemplo, data da edição e aspectos demográfico do autor (sexo, nacionalidade, etc.).

Para a recomendação de objetos de aprendizagem, a exemplo de [24], os metadados Tipo de Interatividade e Tipo de Recurso de Aprendizagem da categoria Educacional do padrão LOM poderiam ser utilizados como MOs.

Retomando a definição do perfil do usuário (2), o conjunto de documentos acessados por um usuário $\mathrm{u}_{\mathrm{k}}$ é definido por:

$$
C A_{k}=\left\{d_{p} \mid \mathrm{p} \in\left[1, \mathrm{D}_{\mathrm{k}}\right]\right\}
$$

onde $\mathrm{d}_{\mathrm{p}}$ é um conjunto de documentos acessados pelo usuário $u_{\mathrm{k}}$ e $\mathrm{D}_{\mathrm{k}}$ é o número total de conteúdos acessados por este usuário.

As Preferências Específicas de um usuário $u_{k}\left(\mathrm{PE}_{\mathrm{k}}\right)$ são definidas por (5).

$$
P E_{k}=\left\{S V_{k, n} \mid \mathrm{n} \in[1, \mathrm{~N}]\right\}
$$

onde $\mathrm{N}$ denota o número de $\mathrm{MOs}, \mathrm{SV}_{\mathrm{k}, \mathrm{n}}$ é um conjunto de valores ponderados do metadado observado $\mathrm{mo}_{\mathrm{n}}$ construído a partir dos conteúdos acessados pelo usuário $u_{\mathrm{k}}$. Neste conjunto, cada valor de $\mathrm{mo}_{\mathrm{n}}$ está associado a um 
peso de preferência $\left(\mathrm{w}_{\mathrm{k}, \mathrm{n}}\right)$ que pode variar de 0 a 1 . Assim, $\mathrm{SV}_{\mathrm{k}, \mathrm{n}}$ é representado como um conjunto de valores ponderados, como definido em (6).

$$
S V_{k, n}=\left\{v w_{k, v} \mid \mathrm{v} \in\left\lfloor 1, \mathrm{~V}_{\mathrm{k}, \mathrm{n}}\right\rfloor\right\}
$$

onde $\mathrm{V}_{\mathrm{k}, \mathrm{n}}$ é o número de valores distintos do $\mathrm{mo}_{\mathrm{n}}$ encontrados nos conteúdos acessados pelo usuário $\mathrm{u}_{\mathrm{k}}$; $\mathrm{vw}_{\mathrm{k}, \mathrm{v}}$ é o valor ponderado $\left(\mathrm{v}_{\mathrm{k}, \mathrm{v}}, \mathrm{w}_{\mathrm{k}, \mathrm{v}}\right)$, onde $\mathrm{v}$ é um valor do metadado $m_{\mathrm{n}}$ e $\mathrm{w}_{\mathrm{k}, \mathrm{v}} \in[0,1]$ representa o peso de preferência deste valor para o usuário $u_{k}$. Quanto maior for esse peso, maior será o interesse do usuário para este valor de elemento específico.

Os conjuntos de valores ponderados dos elementos de metadados observados (SV) são atualizados quando o usuário acessa um novo documento. Quando o usuário $u_{k}$ acessa um documento $\mathrm{d}_{\mathrm{i}}$, cada valor do elemento de $\mathrm{MO}$ tem seu peso de preferência incrementado. Por exemplo na BD-LB, quando um usuário acessa um conto escrito por José de Alencar, o peso de preferência do valor "José de Alencar" em SV $\mathrm{V}_{\text {k.autor }}$ e o peso de preferência do valor “conto" em $\mathrm{SV}_{\mathrm{k}, \text { gênero }}$ devem ser incrementados.

Com base em [29], foram identificadas três análises básicas utilizadas para incrementar o peso de preferência dos valores de MOs:

- Análise de Existência: o peso de preferência de um valor de MO pode assumir os valores 0 e 1 . Caso o usuário não tenha acessado documentos com este valor de $\mathrm{MO}$, o peso será 0 , senão será 1 . Note que neste modo a quantidade de acesso a documentos com o valor de MO não é considerado.

- Análise de Frequência: o peso de preferência associado com um valor de MO é definido pela frequência de ocorrência deste valor nos documentos acessados. Ou seja, é considerado o número de vezes que este valor ocorre nos documentos acessados. Existem ao menos dois métodos possíveis para estimar o peso de preferência: Whole-History (WH), o peso de preferência é definido pelo número total de acessos; e o método Past-Days (PD), onde serão considerados apenas os valores dos MOs encontrados nos documentos acessados pelo usuário nos últimos $n$ dias.

- Análise de Idade de Acesso: esta análise considera que os acessos mais recentes são mais relevantes para considerar a preferência do usuário do que os acessos mais antigos. Em [29], os autores propõem uma equação para calcular o peso baseado na idade acesso.

A técnica proposta independe do tipo de análise utilizada para determinar o peso de preferência do valor do MO. Esta escolha dependerá do tipo de conteúdo oferecido pelo RD. Algumas considerações devem ser realizadas na escolha da análise:

- A análise de existência é simplista e só poderia ser adotada em casos em que a simples ocorrência do valor de MO em um dos conteúdos acessados possa indicar que o usuário tem preferência por conteúdos com este valor. Por ser um valor binário, não é possível diferenciar preferências entre estes valores. Além disso, ela é válida apenas em situações em que as preferências do usuário não alteram ao longo do tempo;

- A análise de frequência pode ser aplicada quando as preferências do usuário não mudam ao longo do tempo (na WH), ou são preferências que se mantém por um determinado período de tempo (na PD).

- A análise de idade de acesso é útil quando as preferências do usuário se alteram ao longo do tempo.

No caso do domínio da literatura, considerou-se empiricamente que as preferências do usuário não se alteram muito ao longo do tempo. Mas consideramos que testes devem ser realizados para verificar a validade desta hipótese. Devido a esta consideração, neste trabalho foi adotado, a título de exemplo, o uso da análise WH para determinar o peso de preferência. Sendo assim, o peso de preferência de um valor de metadado mon para um usuário $\mathrm{u}_{\mathrm{k}}$ é definido por (7).

$w_{k, v}=\operatorname{numAcessos}\left(m o_{n}, v\right) / D_{k}$

onde numAcessos $\left(m o_{n}, v\right)$ é o número de documentos acessados com o valor do elemento de metadados mon igual a $\mathrm{v}$, e $\mathrm{D}_{\mathrm{k}}$ é o número total de documentos acessados pelo usuário $\mathrm{u}_{\mathrm{k}}$.

Para ilustrar os conceitos apresentados, considere o repositório $\mathrm{BD}-\mathrm{LB}$ e os $\mathrm{MOs}$ : autor $\left(\mathrm{mo}_{\text {autor }}\right)$ e gênero literário (mogênero). Considere também três usuários do repositório $\mathrm{u}_{1}, \mathrm{u}_{2}, \mathrm{e} \mathrm{u}_{3}$, que acessaram cada um deles 10 documentos distintos da BD-LB, sendo que:

- $\mathrm{u}_{1}$ acessou 7 obras de "Machado de Assis" e 3 obras de "José de Alencar", sendo que 8 destas obras são do gênero "Conto" e 2 obras são do gênero "Romance".

- $\mathrm{u}_{2}$ acessou 4 obras de "Gregório de Matos" e 6 obras de "Machado de Assis", sendo que 5 destas obras são do gênero "Poesia" e 5 obras são do gênero "Conto".

- $\mathrm{u}_{3}$ acessou 3 obras de "Machado de Assis", 2 obras de "Aluízio Azevedo", 3 obras de "José de Alencar" e 2 obras de "Gregório de Matos", sendo que 3 destas obras são do gênero "Conto", 2 obras são do gênero "Romance", 2 obras são "Poesia" e 3 são do gênero "Teatro". 
De acordo com (5) a (7), as PE destes usuários são:

- $\mathrm{PE}_{1}=\{\{($ Machado de Assis, 0.7), (José de Alencar, 0.3) $\}\{($ Conto, 0.8), (Romance, 0.2$)\}\}$

- $\mathrm{PE}_{2}=\{\{($ Gregório de Matos, 0.4), (Machado de Assis, 0.6) $\}$ (Poesia, 0.5), (Conto, 0.5) $\}$ \}

- $\mathrm{PE}_{3}=\{\{($ Machado de Assis, 0.3), (Aluízio Azevedo, 0.2), (José de Alencar, 0.3), (Gregório de Matos, 0.2)\} \} \{(Conto, 0.3), (Romance, 0.2), (Poesia, 0.2), (Teatro, 0.3) $\}$ \}

$\mathrm{O}$ peso de preferência associados aos valores dos elementos de metadados são atualizados sempre que um usuário acessa um novo conteúdo, usando a análise na frequência. Por exemplo, se o usuário $\mathrm{u}_{1}$ acessar um novo romance de "José de Alencar", $\mathrm{PE}_{1}$ passaria para $\{\{(\mathrm{Ma}-$ chado de Assis, 7/11), (José de Alencar, 4/11) $\}\{($ Conto, 8/11), (Romance, 3/11)\}\}.

\subsection{Agrupamento de Usuários}

A próxima etapa no processo de recomendação é a FC. Para tal, se faz necessária a descoberta dos vizinhos próximos. Visando a redução do tempo de determinação dos vizinhos próximos em RDs com grande número de usuários, foi definida uma técnica de agrupamento de usuários. Nesta técnica, cada grupo reúne usuários com interesses similares, chamados de grupos de interesse.

O processo de agrupamento se dá pela análise dos perfís dos usuários do RD. A determinação dos grupos de interesse a que um determinado usuário será adicionado depende do peso de preferência de determinados valores de MOs. Quando um determinado valor de MO atingir um limiar de peso de preferência, o usuário será incluído no grupo de interesse deste valor. Este limiar é chamado de limiar de agrupamento.

O conjunto de agrupamentos de usuários de um repositório (G) é definido por (8).

$G=\left\{g_{m o_{a}, v_{q}} \mid \mathrm{a} \in[1, \mathrm{~A}], q \in\left[1, \mathrm{~V}_{\mathrm{n}}\right]\right\}$

Sendo que:

- A é o número de MOs considerados para agrupamento;

- $\mathrm{V}_{\mathrm{n}}$ é o número de valores distintos do $\mathrm{mo}_{\mathrm{n}}$.

$\mathrm{Na}$ equação (8) observa-se que o número de grupos dependerá do número de MOs considerados para agrupamento e também do número de valores distintos destes MOs. Portanto, diferente do $k$-means, o número de grupos é dependente da alta frequência de diferentes valores de metadados dos perfis dos usuários. Quanto mais dispersos são os perfis, mais grupos serão criados.
O Algoritmo 1 especifica a técnica de agrupamento proposta. Este algoritmo é executado periodicamente (p.e., a cada 24h) para atualização dos grupos. Inicialmente o conjunto de grupo $\mathrm{G}$ é vazio. Em seguida, para cada perfil de usuário do RD é analisado o peso de cada um dos valores dos MOs. Caso o valor de um destes metadados atingir um limiar de agrupamento, o usuário será incluído no grupo identificado por este valor. Por exemplo, se o usuário realiza muitos acessos a obras de determinado autor ou gênero literário, o usuário será incluído no grupo deste autor e no grupo deste gênero literário.

O valor do limiar de agrupamento é específico do MO. Seu valor depende principalmente da quantidade de valores distintos possíveis dos metadados observado. Por exemplo, os metadados observados da BD-LB são autores e gêneros literários. $\mathrm{O}$ número de autores possíveis atualmente é na ordem de milhares, enquanto os gêneros possíveis são 23 valores. Quando o elemento de metadado tem muitos valores possíveis, como autor, o limiar de agrupamento poderia ser menor, devido à possibilidade de maior dispersão dos usuários. A título de ilustração, considere o MO de agrupamento Autor, os usuários $\mathrm{u}_{1}, \mathrm{u}_{2}$ e $u_{3}$ e um limiar de agrupamento comum de 0,4 (40\%). A Tabela 1 reapresenta os pesos de preferência dos valores do MO Autor para os usuários considerados.

Considerando o limiar de agrupamento de 0,4 , serão criados os grupos do metadado Autor apresentados na Tabela 2. Estes grupos foram determinados aplicando o algoritmo de agrupamento descrito na Figura 1. Note que $\mathrm{o}$ usuário $\mathrm{u}_{3}$ não será incluído em nenhum grupo de $\mathrm{MO}$ Autor. Considera-se que o perfil deste usuário não permite ainda determinar as suas preferências em termos de autores. Consequentemente, a FC não permitirá recomendar obras para $u_{3}$. Ele receberá recomendações com base na FBC (Seção 4.6).

\begin{tabular}{l|l|l}
\hline Usuário & \multicolumn{1}{|c|}{ Autor } & Preferência \\
\hline u1 & Machado de Assis & 0.7 \\
\hline u1 & José de Alencar & 0.3 \\
\hline u2 & Gregório de Matos & 0.4 \\
\hline u2 & Machado de Assis & 0.6 \\
\hline u3 & Machado de Assis & 0.3 \\
\hline u3 & Aluízio Azevedo & 0.2 \\
\hline u3 & José de Alencar & 0.3 \\
\hline u3 & Gregório de Matos & 0.2
\end{tabular}

Tabela 1: Pesos de Preferência dos valores do MO Autor para os usuários $\mathrm{u}_{1}, \mathrm{u}_{2}$ e $\mathrm{u}_{3}$

\begin{tabular}{l|l}
\hline \multicolumn{1}{c|}{ Grupo Autor } & Usuários \\
\hline Machado de Assis & ul, u2 \\
\hline Gregório de Matos & $\mathrm{u} 2$
\end{tabular}

Tabela 2: . Grupos de $M O$ Autor e seus usuários 


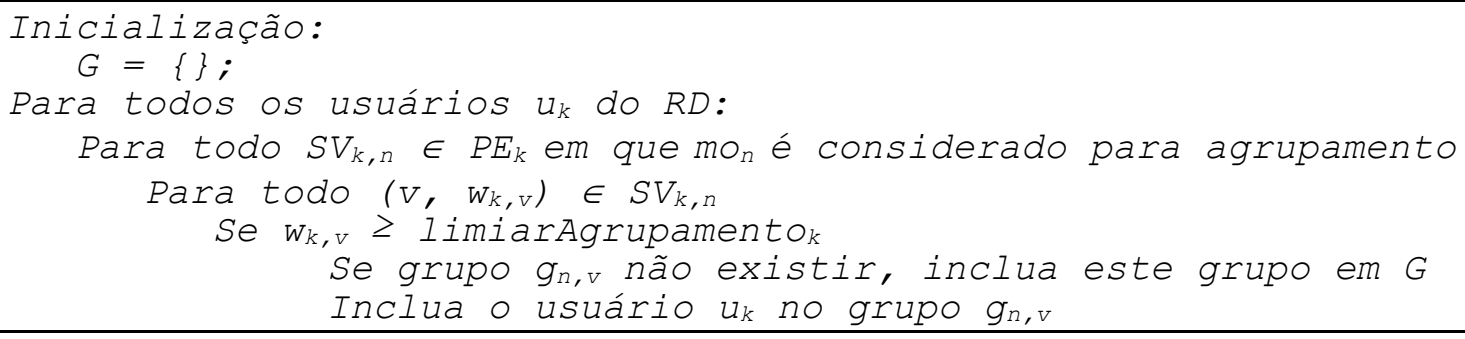

Figura 1: Algoritmo de Agrupamento

Um aspecto crítico nesta proposta é a determinação de quais MOs serão considerados para o agrupamento. No contexto da BD-LB, considerou-se autor e gênero literário como metadados de agrupamento, pois tais características são muito relevantes para levar um usuário a preferir uma determinada obra literária. Como contraexemplo, um metadado como idioma seria inapropriado para o agrupamento, pois se um usuário acessar muitas obras de determinado idioma, não quer dizer necessariamente que ele se interessaria por qualquer conteúdo em tal idioma.

$\mathrm{Na}$ técnica de agrupamento proposta, a lógica simples de agrupamento e criação de grupos não padroniza o número de grupos ou similaridade de tamanho. Podendo haver grupos com 1 usuário, e grupos com um grande número de usuários. A vantagem desta técnica de agrupamento se dá, além do processamento reduzido do algoritmo de recomendação aplicado a um grupo, pelo fato de que usuários com tais características de alta frequência de ocorrência de valores de MOs têm maior grau de similaridade entre si. Dessa forma, o algoritmo de FC, ao explorar apenas os usuários de determinado grupo, tenderá a localizar usuários com maior grau de similaridade entre si, sem a necessidade de explorar outros usuários que tenderão a ter pouca (ou nenhuma) relação com os usuários do grupo pesquisado. Assim, a técnica resulta em um menor custo de processamento, sem no entanto prejudicar a qualidade da recomendação.

A técnica proposta pode ser facilmente estendida para que um grupo de interesse possa considerar ao mesmo tempo dois ou mais metadados de agrupamento. Por exemplo, um grupo de interesse pode agrupar usuários preferindo determinado autor e determinado idioma.

\subsection{Determinação dos vizinhos próximos}

Nesta proposta, "vizinhos próximos" a um usuário $u_{k}$ são os usuários que têm uma similaridade de perfil com o usuário $u_{\mathrm{k}}$ acima de certo valor, chamado aqui de limiar de similaridade. O valor de tal limiar dependerá do domínio do RD e dos metadados observados. No caso da BD$\mathrm{LB}$, após a realização de testes, definiu-se empiricamente o valor de 0,7 como limiar de similaridade. Os vizinhos próximos de um usuário $\mathrm{u}_{\mathrm{k}}$, notado por $\mathrm{VP}_{\mathrm{k}}$, é definido por (9).

$V P_{k}=\left\{u_{v p} \mid \operatorname{sim}_{\text {perfil }}\left(\mathrm{pu}_{\mathrm{k}}, \mathrm{pu}_{\mathrm{vp}}\right) \geq \operatorname{limiar}\right\}$

onde $\mathrm{u}_{\mathrm{vp}}$ é um outro usuário que pertence a pelo menos um grupo que $\mathrm{u}_{k}$ faz parte, $\operatorname{sim}_{\text {perfil }}$ é a função que determina o grau de similaridade entre dois perfis de usuário, e limiar é o limiar de similaridade utilizado.

A determinação da similaridade dos perfis dos usuários é realizada em duas etapas. Na primeira é determinada a similaridade entre os conjuntos SVs (conjunto de valores ponderados dos metadados observados) contidos nos perfis de $u_{k} e u_{v p}$.

Para o cálculo de similaridade entre SVs de um metadado observado foi adotada a função do cosseno. Muitas técnicas de FC fazem uso de cálculos como o coeficiente de Pearson para determinar um grau de similaridade entre usuários. O coeficiente de Pearson é usado para determinar uma correlação entre duas variáveis (perfis de usuários) e fornece um resultado variando entre +1 e -1 . Nela, +1 significa total correlação, 0 nenhuma correlação e -1 total correlação negativa. Nesta proposta, na etapa de identificação de usuários similares, busca-se encontrar similaridades entre perfis de usuários, sem haver uma correlação negativa. Dessa forma, foi adotada a fórmula do cosseno para calcular a similaridade, que fornece resultados entre 0 (nenhuma relação entre os perfis) e +1 (perfis idênticos). Portanto, a similaridade entre os conjuntos SVs de um metadado observado $\mathrm{mo}_{\mathrm{n}}$ é determinada por (10).

$$
\operatorname{sim}_{\mathrm{mo}_{\mathrm{n}}}\left(\mathrm{pu}_{\mathrm{k}}, \mathrm{pu}_{\mathrm{vp}}\right)=\frac{\sum_{v=1}^{V_{k, n}} w_{k, v} \cdot w_{v p, v}}{\sqrt{\sum_{v=1}^{V_{k, n}}\left(w_{k, v}\right)^{2} \cdot \sum_{v=1}^{V_{k, n}}\left(w_{v p, v}\right)^{2}}}
$$

onde $\mathrm{pu}_{\mathrm{k}}$ representa o perfil do usuário foco da recomendação $\left(u_{k}\right) ; u_{v p}$ representa o perfil de um vizinho próximo do grupo a ser comparado $\left(\mathrm{u}_{\mathrm{vp}}\right) ; \mathrm{V}_{\mathrm{k}, \mathrm{n}}$ é o número de valores distintos do elemento de metadado $\mathrm{mo}_{\mathrm{n}}$ descrevendo os documentos acessados por $\mathrm{u}_{\mathrm{k}}$; $\mathrm{w}_{\mathrm{k}, \mathrm{v}}$ é o peso de preferência do valor $\mathrm{v}$ do elemento de metadados mon $_{n}$ para o usuário $\mathrm{u}_{\mathrm{k}}$; $\mathrm{w}_{\mathrm{vp}, \mathrm{v}}$ é o peso de preferência do valor 
de $\mathrm{v}$ do elemento de metadados $\mathrm{mo}_{\mathrm{n}}$ se presente no perfil do $\mathrm{u}_{\mathrm{vp}}$ (senão, $\mathrm{w}_{\mathrm{vp}, \mathrm{v}}$ é zero).

A título de exemplo, considere a recomendação realizada para o usuário $\mathrm{u}_{1}$. Os usuários $\mathrm{u}_{1}$ e $\mathrm{u}_{2}$ terão seus perfis comparados, pois ambos estão contidos nos grupos $\mathrm{G}_{\text {Autor,Machado de Assis; }}$ e $\mathrm{G}_{\text {Gênero,Conto. Nesta etapa é calculada }}$ a similaridade dos metadados autor e gênero: $\operatorname{sim}_{\text {autor }}(1,2)$ $=0,76$; e sim $\operatorname{senero~}(1,2)=0,69$. Em seguida, para determinação da similaridade geral, é calculada a média simples entre as similaridades de cada MO, conforme Equação (11).

$\operatorname{sim}_{p e r f i l}\left(p u_{k}, p u_{v p}\right)=\frac{\sum_{n=1}^{N} \operatorname{sim}_{m o_{n}}\left(p u_{k}, p u_{v p}\right)}{N}$

onde $\mathrm{N}$ é o número de metadados usado para agrupamento.

Usando (11), a similaridade dos perfis de $\mathrm{u}_{1}$ e $\mathrm{u}_{2}$ resultaria em $\operatorname{sim}_{\text {perfil }}(1,2)=0.73$. Lembrando que, com a adoção do agrupamento dos usuários, $\mathrm{u}_{1}$ e $\mathrm{u}_{3}$ não serão comparados por não possuírem nenhum grupo em comum, visto que $u_{3}$ não foi incluído nem no grupo $\mathrm{G}_{\text {Autor,Machado de }}$ Assis, nem em $\mathrm{G}_{\text {Gênero,Conto. }}$

\subsection{Construção da Recomendação}

Esta etapa final tem por objetivo produzir a lista ordenada de conteúdos a serem recomendados a um determinado usuário. Esta lista é obtida a partir do conjunto de conteúdos acessados pelos vizinhos próximos do usuário foco da recomendação e que ainda não foram acessados pelo usuário. Desta forma, estes conteúdos já acessados pelo usuário serão retirados da lista de recomendação.

A lista de recomendação é ordenada com base no cálculo do peso de preferência definido por [27]. Assim, os conteúdos que tiverem maior chance de serem interessantes para o usuário aparecem primeiro. Desta forma, a lista ordenada de conteúdos recomendados $\mathrm{LR}_{\mathrm{k}}$ para um usuário $\mathrm{u}_{\mathrm{k}}$ é definida em (12).

$$
\begin{aligned}
& L R_{k}=\left(d_{1}, d_{2}, \ldots, d_{R}\right) \text { onde } \\
& \forall r \in[1, R-1], w_{p r e f}\left(u_{k}, d_{r}\right) \geq w_{p r e f}\left(u_{k}, d_{r+1}\right)
\end{aligned}
$$

$\mathrm{O}$ peso de preferência $\mathrm{w}_{\text {pref }}$ de um conteúdo $\mathrm{d}_{\mathrm{r}}$ a ser recomendado é estimado pela combinação dos pesos de preferência de cada um dos valores dos elementos de MOs deste conteúdo. Note aqui que é utilizado a FBC, pois agora a técnica está se apoiando nas características dos conteúdos (como descritos pelos seus metadados).

Para exemplificar o cálculo do peso de preferência, considere novamente o usuário $\mathrm{u}_{1}$ da BD-LB com seu perfil descrito na seção 4.2, e a lista de conteúdos a serem recomendados constantes na Tabela 3. Estes documentos foram supostamente acessados pelos vizinhos próximos de $u_{1}$.

De acordo com as PEs de $u_{1}$ (apresentado na seção 4.2), o valor de preferência de cada conteúdo será calculado como a média entre o peso de preferência dos valores dos elementos de metadado autor e gênero. O resultado deste cálculo também é apresentado na Tabela 3. Finalmente, com base no peso de preferência calculado, a lista de conteúdo pode ser ordenada, conforme apresentado na Tabela 3 .

\subsection{Tratando a esparsidade}

Na técnica proposta até aqui, a lista de recomendação como calculada na seção anterior só poderá conter conteúdos que já foram acessados pelos vizinhos próximos do usuário foco da recomendação. Para reduzir este problema de esparsidade, propõe-se aqui a utilização da técnica de FBC. Neste caso, a lista de recomendação obtida na FC será complementada com a lista de recomendação obtida na FBC, caso o número de conteúdos recomendados seja inferior ao número desejado (definido na aplicação da técnica).

A técnica de FBC adotada aqui é a mesma apresentada na seção 4.5 , exceto que ela não considera apenas os conteúdos acessados pelos vizinhos próximos do usuário, mas considera todos os conteúdos cadastrados no RD. Para aumento da escalabilidade da FBC, é necessário a construção de uma tabela itens-similares, calculada de maneira off-line, similar ao proposto pela Amazon.com [16]. Mas diferente deste último, em vez de considerar itens similares aqueles que os clientes tendem a comprar junto, a presente proposta agrega itens similares aqueles que têm os mesmos valores nos metadados observados.

\begin{tabular}{l|l|l|c|c|c}
\hline \multicolumn{1}{c|}{ Título } & \multicolumn{1}{|c|}{ Autor } & Gênero & wautor & W $_{\text {gênero }}$ & W $_{\text {pref }}$ \\
\hline Casa velha & Machado de Assis & Romance & 0,7 & 0,2 & 0,45 \\
\hline A paixão & Manuel de Paiva & Conto & 0 & 0,8 & 0,4 \\
\hline Niterói & José de Alencar & Poesia & 0,3 & 0 & 0,15
\end{tabular}

Tabela 3: Lista de Recomendação para o usuário $u_{1}$ 


\section{Implementação e testes realizados}

Esta seção apresenta uma visão geral da implementação da técnica de recomendação proposta no repositório digital BD-LB. Com o objetivo de avaliar a técnica proposta, foi realizado um conjunto de testes a fim de verificar a sua eficiência e tempo de resposta.

\subsection{Implementação na BD-LB}

A BD-LB contém uma vasta coleção de obras literárias de domínio público e informações sobre escritores brasileiros. Atualmente estão catalogadas 74.759 obras de 18.580 autores. Das obras cadastradas, a BD-LB conta com 4.331 arquivos digitalizados, sendo que 1.690 são obras literárias de domínio público. A plataforma é caracterizada pelo uso de código aberto, adotando-se LAMP (Linux, Apache, MySQL, PHP).

A BD-LB permite o cadastramento dos usuários e o sistema estima implicitamente o perfil do usuário como apresentado neste trabalho. A recomendação de obras é realizada na página principal da BD-LB após o usuário realizar a autenticação. Esta lista de recomendação é obtida usando a técnica proposta neste artigo.

A BD-LB adota um conjunto de metadados para descrever os conteúdos disponibilizados. Como já citado, foram adotados dois metadados para compor o perfil do usuário (metadados observados): "Autor" e "Gênero Literário". Nesta implementação foi adotado como limiar de agrupamento o valor 0,4 tanto para o metadado autor quanto para o metadado gênero. O limiar de similaridade adotado foi de 0,7 .

O processo de agrupamento dos usuários (Seção 4.3) e atualização da tabela itens-similares (Seção 4.6) é realizado a cada 24 horas, durante o período de menor utilização do servidor. O processo de determinação dos vizinhos próximos e determinação da lista de recomendação (Seções 4.4 e 4.5) são executados no momento em que o usuário cadastrado acesse a página principal (que apresenta a lista de recomendação).

\subsection{Testes}

Foram realizados três tipos de avaliações da técnica proposta: cobertura, precisão e tempo de resposta.

\subsubsection{Cobertura de Itens e de Usuários}

Existem duas métricas importantes utilizadas para analisar sistemas de recomendação, que são a cobertura de item e de usuários [30]. A cobertura de itens se refere a proporção de itens que o sistema pode recomendar. Por sua vez, a cobertura de usuários se refere a possibilidade de se realizar recomendações a determinados usuários. Esta última pode ser medida pela riqueza do perfil do usuário requerida para realizar a recomendação. Para avaliar a cobertura de itens e usuários, foi utilizada a BDLB com suas obras literárias e usuários reais.

$\mathrm{O}$ sistema de recomendação baseado na $\mathrm{FC}$ só pode recomendar itens que já foram acessados por pelo menos um usuário. Considerando $\mathrm{I}_{\mathrm{a}} \mathrm{o}$ número de itens acessados pelo conjunto de usuários e $\mathrm{I}_{\mathrm{t}} \mathrm{o}$ total de itens do $\mathrm{RD}$, a cobertura de itens seria dada por $\mathrm{I}_{a} / \mathrm{I}_{\mathrm{t}}$. Atualmente, a BDLB conta com 438 usuários que habilitaram o sistema de recomendação, sendo que estes mesmos usuários acessaram 316 obras distintas de um total de 1.690 obras recomendáveis. Portanto, a cobertura de itens seria baixa $(18,7 \%)$ se o sistema utilizasse apenas FC. Com a combinação da FBC, a cobertura de itens alcança $100 \%$, pois todas as obras são consideradas na recomendação. A perda de desempenho da FBC foi reduzida com a criação da tabela itens-similares, que na situação atual da BD-LB reduziu o número de obras de 1.690 para 1.384 obras similares (redução de 18,1\%).

Em relação a cobertura de usuários, se considerada apenas a FC, o usuário apenas receberia a recomendação após ele pertencer a um grupo de interesse com outros usuários e que estes usuários sejam seus vizinhos próximos. Atualmente, os 438 usuários são agrupados em 19 grupos do metadado gênero e 60 grupos do metadado autor. Sendo que 21 destes grupos apresentam apenas um usuários, portanto, se considerada apenas a $\mathrm{FC}$, atualmente $4,8 \%$ dos usuários não receberiam a recomendação via a FC. Mas com a combinação da FBC (Seção 4.6), todos os receberão recomendação (desde que ele tenha acessado ao menos uma obra).

\subsubsection{Teste de Precisão}

Além da cobertura, as métricas de precisão (precision) e revocação (recall) são usadas para avaliar sistemas de recomendação. A técnica de precisão é medida pela razão entre o número de documentos relevantes recuperados e o número total de documentos recuperados pelo sistema. Já a revocação é definida pela relação entre o número de documentos relevantes recuperados e o número total de documentos relevantes contidos no sistema. Para avaliar a técnica de recomendação proposta, foi utilizada apenas a métrica de precisão. Não foi possível utilizar a métrica de revocação devido ao grande número de obras disponíveis. Para que esta métrica fosse utilizada, todos os usuários do experimento teriam que avaliar a relevância de todas as obras disponíveis.

Para realizar a medida da precisão, foi realizado um experimento com 24 usuários reais da BD-LB. Inicialmente os participantes acessaram 5 obras de sua preferên- 
cia. Em seguida, foi informado para cada usuário uma lista de 5 obras recomendadas. Os participantes então avaliaram cada obra com notas de 1 a 5 de acordo com seu nível de relevância para leitura. Para fins de cálculo da precisão, considerou-se que o conteúdo é relevante se a nota dada pelo usuário é superior ou igual a 3 . Além disso, devido à baixa quantidade de usuários, adotou-se o limiar de similaridade de 0,5 .

$\mathrm{O}$ índice de precisão médio obtido neste experimento foi de 0,78 com desvio padrão de 0,25 . A precisão tende a aumentar de acordo com o aumento do número de usuários. Para verificar isso, foi realizado anteriormente um teste similar com apenas 6 usuários, onde a precisão média foi de 0,625 com desvio padrão de 0,14 .

\subsubsection{Tempo de Processamento}

Esta seção apresenta os testes para medir o tempo de processamento da técnica proposta, visando medir a escalabilidade do sistema proposto. Para a realização deste teste foram inseridos 10.000 usuários fictícios no repositório e, para cada um deles, foi simulado o acesso a 10 obras dentre um conjunto de 748 obras. Para os testes, foi utilizado um servidor não dedicado com processador Intel Quad-Core Xeon E5405 2GHZ, com 8GB de memória.

Na seleção das 10 obras acessadas pelos usuários, tentou-se emular o comportamento dos usuários reais, considerando a existência de autores populares, e também que geralmente usuários tendem a ler um pequeno conjunto de autores. Autores mais populares são considerados aqui aqueles mais acessados por mais usuários. Para emular esse padrão, o acesso a 10 obras simulado no teste não foi totalmente aleatório: para cada usuário, as seis primeiras obras foram limitadas a dois autores escolhidos aleatoriamente pertencentes ao grupo dos 15 autores mais populares do repositório, sendo 3 acessos para cada um dos dois autores. Em seguida, os quatro últimos acessos são aleatórios, dentre todo o conjunto de obras digitalizadas.

Para viabilizar a realização do teste, foram escolhidos aleatoriamente 100 dos 10.000 usuários cujos tempos de processamento das recomendações seriam medidos. Para cada um destes usuários foram geradas duas listas de recomendação, cada uma contendo 10 obras recomendadas. Uma das listas foi gerada sem o uso da técnica de agrupamento, e outra com o uso de agrupamento com limiar de agrupamento de 0,4. Para estudo comparativo, foram medidos os tempos de processamento e também as listas de obras recomendadas.

Após a execução do agrupamento de usuários, foram criados 23 grupos ( 15 de autores e 8 de gêneros), sendo o maior contendo 2.521 usuários e o menor com 8 usuários. A média de tamanho dos grupos foi de 414. Agora analisando apenas os 100 usuários cujos tempos de processa- mento foram medidos, 63 foram alocados em grupos, contra 37 que não foram.

A Figura 2 apresenta os tempos de processamento médio das recomendações com e sem agrupamento. Em média, o tempo de processamento da recomendação sem agrupamento foi de $6,68 \mathrm{~s}$, com desvio padrão de $0,43 \mathrm{~s}$. Com a utilização da técnica de agrupamento proposta obteve-se uma média de $3,9 \mathrm{~s}$, com desvio padrão de $2,37 \mathrm{~s}$. Estes resultados mostram que houve um ganho significativo no tempo de processamento com o uso da técnica de agrupamento.

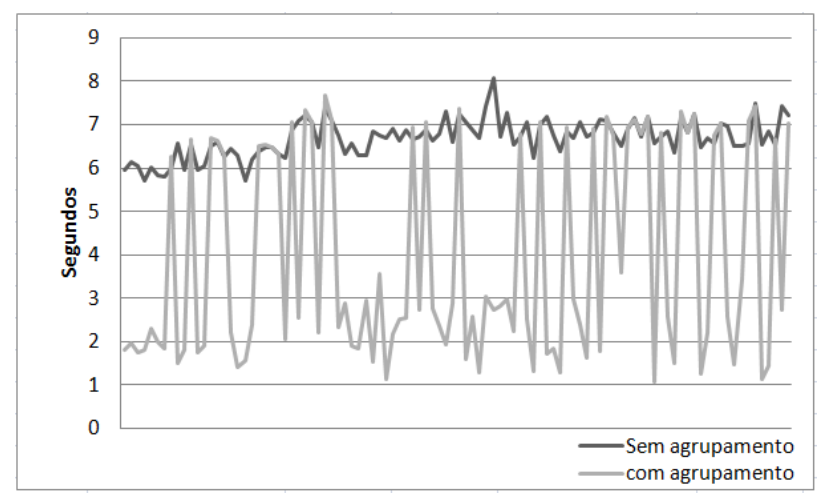

Figura 2: Tempos de processamento médio das recomendação

Pode-se notar na Figura 2 o elevado desvio padrão com o uso do agrupamento. Isto se deve ao fato de alguns usuários não estão incluídos em nenhum grupo (por não alcançarem frequência de acesso superior ao limiar de 0,4). Para tais casos, a FC sem agrupamento é realizada, sem prejudicar o tempo de processamento, já que o tempo necessário para a recomendação será igual ao pior caso, como mostra a Figura 2. Considerando apenas os usuários que pertenciam a grupos, a média do tempo de processamento cai para $2,13 \mathrm{~s}$, com desvio padrão de $0,62 \mathrm{~s}$.

$\mathrm{O}$ processo de agrupamento visa reduzir o espaço de busca da FC a fim de obter um tempo de resposta otimizado. Uma característica negativa deste processo se dá pela possível redução da qualidade da recomendação. Para avaliar o impacto na qualidade da recomendação, foi realizado um estudo comparativo de qualidade entre duas listas de recomendação: uma utilizando a técnica de agrupamento, e a outra não. Tais listas foram comparadas entre si para que pudesse ser avaliado o percentual de perda de qualidade entre as recomendações.

Dos 100 usuários analisados, todas as recomendações geradas com e sem a técnica de recomendação eram idênticas. Portanto, neste teste realizado, a aplicação da técnica de agrupamento proposta não resultou em nenhuma perda de qualidade. Isso não significa que a proposta é livre de perda de qualidade, podendo ocorrer em outros cenários de teste. A baixa, ou nenhuma, perda de qualida- 
de pode ser justificada pelo cálculo do cosseno da FC utilizada que define os vizinhos próximos. Nele, usuários que tenham altas frequências $(>0,4)$ de acesso ao mesmo valor de metadado terão alto valor no grau de similaridade entre si. Isto faz com que a grande maioria dos vizinhos próximos de um determinado usuário esteja situada nos mesmos grupos que o usuário em questão esteja alocado, tornando desnecessária a busca por vizinhos próximos em toda a lista de usuários cadastrados no sistema.

\section{Conclusões}

Este artigo propôs uma técnica de recomendação de conteúdos para repositórios digitais, considerando a existência de metadados que descrevem os conteúdos. O objetivo desta técnica é reduzir o esforço despendido pelo usuário na localização de conteúdos relevantes.

A técnica proposta, diferente das existentes, analisa valores dos elementos de metadado dos conteúdos acessados pelos usuários a fim de estimar implicitamente suas preferências. $\mathrm{O}$ conjunto de elementos de metadado a ser observado é dependente do domínio do repositório, permitindo que metadados descritores de objetos de aprendizagem sejam considerados.

A técnica de recomendação proposta é híbrida, combinando a FC e a FBC. Em ambas as filtragens, são aplicadas técnicas de agrupamento de usuários com interesses comuns (na FC) e de conteúdos similares (na FBC). O agrupamento de usuários permite reduzir o espaço de busca por vizinhos próximos na FC. Assim, é possível criar um sistema de recomendação para RDs com menor custo computacional e com perda muito reduzida da qualidade da recomendação. $\mathrm{O}$ mesmo ocorre com o agrupamento de conteúdos similares. Assim, a adoção das técnicas de agrupamento permite tratar parcialmente o problema da escalabilidade.

A efetividade da técnica proposta foi comprovada via avaliação empírica no domínio de obras literárias. Apesar do reduzido número de usuários, os resultados obtidos em termos de precisão e tempos de resposta demonstram a efetividade da técnica proposta, pelo menos no domínio de literatura.

Como trabalho futuro, pretende-se investigar outros métodos para aumentar a escalabilidade, como utilização da SVD (Singular Value Decomposition) [31] e filtragem demográfica. Também pretende-se levar em conta a localização de conteúdos ou domínios administrativos, no caso de repositórios digitais federados.

\section{Agradecimentos}

Este trabalho foi desenvolvido no contexto do projeto
PRONEX Autores, obras e acervos literários catarinenses em meio digital, financiando pela FAPESC-CNPq. O autor Marcus Fillipi Rosso Casagrande foi financiado com uma bolsa de mestrado da CAPES.

\section{Referências}

[1] IEEE Standard Association, IEEE LTSC Working Group 12: Learning Object Metadata, http://ltsc.ieee.org/wg12/index.html, 2015.

[2] Dublin Core Metadata Initiative (DCMI), DCMI Home: Dublin Core Metadata Initiative, http://dublincore.org, 2015.

[3] M. F. R. Casagrande, G. K. da Silva, e R. Willrich. Técnica de Recomendação Baseada em Metadados para Repositórios Digitais Voltadas ao Ensino. In Anais do $24^{\circ}$ Simpósio Brasileiro de Informática na Educação, páginas 677-686, 2013.

[4] R. Burke, e M. Ramezani, M. Matching recommendation technologies and domains, Recommender Systems Handbook. Springer, New York, 2010.

[5] E. Costa, J. Aguiar, e J. Magalhães. Sistemas de Recomendação de Recursos Educacionais: conceitos, técnicas e aplicações. In: II Jornada de Atualização em Informática na Educação (JAIE). II Congresso Brasileiro de Informática na Educação (CBIE), páginas 57-78, 2013.

[6] F. Ricci, L. Rokach, B. Shapira, P. B. Kantor. Recommender Systems Handbook. Springer, New York, 2011.

[7] S. C. Cazella, M. A. S. N. Nunes, E. Reategui. A ciência da opinião: estado da arte em sistemas de recomendação. In: Anais do XXX Congresso da SBC Jornada de Atualização da Informática, 2010.

[8] C. Rana, S. K. Jain. Building a Book Recommender System Using Time Vased Content Filtering. WSEAS Transaction on Computers 11(2):27-33, 2012.

[9] B. Cui, X. Chen. An Online Book Recommendation System Based on Web Service. In: Proceedings of the Sixth Int. Conf. On Fuzzy Systems and Knowledge Discovery, páginas 520-524, 2009.

[10] M.R.C. Ferro, H. M. Nascimento Júnior, F. Paraguaçu, E. B. Costa, L. A. L. Monteiro. Um Modelo de Sistema de Recomendação de Materiais Didáticos para Ambientes Virtuais de 
Aprendizagem". In Anais do $22^{\circ}$ Simpósio Brasileiro de Informática na Educação, páginas 810819,2011

[11] M. A. A. Sibaldo, T. B. M. Sales, I. A. A. R. Calado, I. I. Bittencourt, e E. B. Costa, E.B. Mobile GraW: Uma Aplicação para Dispositivos Móveis Baseada em Comunidades Virtuais de Aprendizagem com Suporte A Recomendação". In Anais do Simpósio Brasileiro de Informática na Educação, p. 214-217, 2007.

[12] J. M. Carroll, e M. B. Rosson. The paradox of the active user. Interfacing Thought: Cognitive Aspects of Human-Computer Interaction. MIT Press, páginas 80-111, 1987.

[13] R. Burke. Hybrid Web Recommender Systems. The Adaptive Web. Springer, Berlin/Heidelberg, páginas 377-408, 2007.

[14] B. Sarwar, G. Karypis, J. Konstan, J. Riedl. Recommender systems for large-scale e-commerce: Scalable neighborhood formation using clustering". In Proc. of the Fifth International Conference on Computer and Information Technology, páginas 158-167, 2002.

[15] Q. Li, B. M. Kim.Clustering Approach for Hybrid Recommender System". In Proceedings of the IEEE /WIC International Conference on Web Intelligence (WI’03), páginas 33-38, 2003.

[16] G. Linden, B. Smith, J. York. Amazon.com recommendations: Item-to-item collaborative filtering. IEEE Internet Computing, 4(1):76-80, 2003.

[17] S. Kanetkar, et al. Web-based Personalized Hybrid Book Recomendation Systems. In Proc. of the IEEE International Conference On Advances in Engineering \& Technology Research (ICAETR - 2014), páginas 1-5, 2014.

[18] M. Monnich, M. Spiering. Adding Value to the Library Catalog by Implementing a Recommendation System. D-Lib magazine, 14(5/6), 2008.

[19] C. Yang, et al. CARES: A Ranking-oriented CADAL Recommender System. In: Proceedings of ACM/IEEE-CS, páginas 203-212, 2009.

[20] J. Herlocker, S. Jung, J. G. Webster. Collaborative Filtering for Digital Libraries. Technical Report Oregon State University. http://hdl.handle.net/1957/28103, 2012.

[21] S. C. Cazella, I. C. Chagas, J. L. Barbosa, E. B. Reategui. Um Modelo para Recomendação de Artigos Acadêmicos Baseado em Filtragem Colaborativa Aplicado à Ambientes Móveis. Revis- ta Novas Tecnologias na Educação 7:12-22, 2008.

[22] T. T. Primo, R. M. Vicari, J. M. C. Silva.Rumo ao Uso de Metadados Educacionais em Sistemas de Recomendação. In Anais do Simpósio Brasileiro de Informática na Educação, páginas 4-8, 2010.

[23] M. R. Bez, J. M. C. da Silva, E. R. Santos, T. Primo, A. Bordignon. Projeto OBAA: Uma abordagem com objetos de aprendizagem interoperáveis baseados na web e na televisão digital. Informática na Educação: Teoria e Prática 12(1):119-126, 2009.

[24] L. A. M. Zaina et al. E-LORS: Uma Abordagem para Recomendação de Objetos de Aprendizagem. Revista Brasileira de Informática na Educação 20(1):4-16, 2012.

[25] R. M. Felder, R. Brent. Understanding Student Differences. Journal of Engineering Education 94(1):57-72, 2005.

[26] A. K. Jain, R. C. Dubes. Algorithms for Clustering Data. Prentice Hall, 1988.

[27] C. A. Furtado, R. Willrich, R. Fileto, F.L. Siqueira, S. Tazi. Ordenação Personalizada na Recuperação de Informações em Bibliotecas Digitais. In Anais do Simpósio Brasileiro de Sistemas Multimídia e Web (WebMedia), páginas 218225, 2009.

[28] M. Koolen, et al. Looking for Books in Social Media: An Analysis of Complex Search Requests. In: Proceedings of the 37th European Conference on Information Retrieval, 2015.

[29] M. Chen, A. S. LaPaugh, J. P. Singh. Predicting Category Accesses for a User in a Structured Information Space. In Proc. Of the 25th Int. ACM SIGIR Conference on Research and Development in Information Retrieval, páginas 65-72, 2002.

[30] G. Shani, A. Gunawardana. Evaluating Recommendation Systems. Recommender Systems Handbook. Springer, New York, páginas 257297, 2011.

[31] B. Sarwar, G. Karypis, J. Konstan, J. Riedl. Application of dimensionality reduction in recommender system-a case study (No. TR-00-043). Minnesota Univ Minneapolis Dept of Computer Science, 2000. 